Research Article

\title{
Some Compatible and Weakly-Compatible Four Self-Mapping Results Approach to Nonlinear Integral Equations in Fuzzy Cone Metric Spaces
}

\author{
Saif Ur Rehman $\mathbb{D}^{1},{ }^{1}$ Hawraa Akram Yazbek, ${ }^{2}$ Rashad A. R. Bantan, ${ }^{3}$ \\ and Mohammed Elgarhy $\mathbb{1 D}^{4}$ \\ ${ }^{1}$ Department of Mathematical, Gomal University, Dera Ismail Khan 29050, Pakistan \\ ${ }^{2}$ Doctoral School of Mathematics and Informatics, University of Bordeaux, France \\ ${ }^{3}$ Department of Marine Geology, Faculty of Marine Science, King Abdulaziz University, Jeddah 21551, Saudi Arabia \\ ${ }^{4}$ The Higher Institute of Commercial Sciences, Al Mahalla Al Kubra, Algarbia 31951, Egypt \\ Correspondence should be addressed to Mohammed Elgarhy; m_elgarhy85@sva.edu.eg
}

Received 8 September 2021; Accepted 21 October 2021; Published 10 December 2021

Academic Editor: John R. Akeroyd

Copyright (c) 2021 Saif Ur Rehman et al. This is an open access article distributed under the Creative Commons Attribution License, which permits unrestricted use, distribution, and reproduction in any medium, provided the original work is properly cited.

\begin{abstract}
This paper is aimed at proving some unique common fixed point theorems by using the compatible and weakly-compatible four self-mappings in fuzzy cone metric (FCM) space. We prove the results under the generalized rational contraction conditions in FCM spaces with the help of one self-map are continuous. Furthermore, we prove some rational contraction results with the weaker condition of the self-mapping continuity. Ultimately, our theoretical work has been utilized to prove the existence solution of the two nonlinear integral equations. This is an illustrative application of how FCM spaces can be used in other integral type operators.
\end{abstract}

\section{Introduction}

The theory of fixed-point theory was introduced by Banach [1]. He proved a "Banach contraction principle," which is stated as follows: "A self-mapping on a complete metric space verifying the contraction condition has a unique fixed point (FP)." Later on, many researchers have been generalized this principle in many directions and proved different contractive type FP and common fixed point (CFP) for single-valued and multivalued mappings in the context of metric spaces. Chatterjea [2], Chatterjea [3], and Kannan [4] proved some single-valued contractive type FP theorems. While Ali et al. [5], Covitz and Nadler [6], Daker and Kaneko [7, 8], Khan [9], and Patle et al. [10] proved multivalued contractive type FP and CFP results by using different types of spaces.

Zadeh [11], in 1965, introduced the concept of fuzzy sets. Later on, this concept was used in topology and functional analysis by many researchers. Kramosil and Michalek [12] introduced the notion of fuzzy metric FM space, and they established some basic properties. After that, George and Veeramani [13] presented the stronger form of the FM. Grabiec [14] proved two FP theorems by using the concept of complete and compact FM spaces. Gregori and Sapena [15] established some FP contraction results in the sense of $[13,15]$. Hadzic and Pap [16] proved a FP theorem for multivalued mappings in probabilistic metric spaces and presented applications in FM spaces. Imdad and Ali [17] and Rehman et al. [18] proved some FP theorems in complete FM spaces. Pant and Chauhan [19] established some CFP theorems by using weakly-compatible mappings in menger spaces and FM spaces. Kiyani et al. [20] and Sadeghi et al. [21] proved some results for set-valued contractive type mappings in FM spaces.

The concept of cone metric space (CMS) was proposed by many researchers but it became popular after being redis- 
covered by Huang and Zhang [22]. They proved the convergence properties and FP theorems for nonlinear contractive type mappings. By using the concept of Huang and Zhang [22], many authors have contributed their work to the problems on CMSs. Some of such works can be found in ([23-28]).

In 2015, the notion of fuzzy cone metric space (FCM space) was introduced by Oner et al. [29]. They proved the key attributes of FCM space and a "fuzzy cone Banach contraction theorem for FP" in FCM space. In [30], Rehman and $\mathrm{Li}$ extended and improved a "fuzzy cone Banach contraction theorem" and established some generalizedcontraction results for FP in FCM spaces. Rehman et al. $[31,32]$ proved different contractive type CFP-theorems in FCM spaces. Recently, the concept of weakly compatible self-mappings in FCM spaces was given by Jabeen et al. [33].

This paper is aimed at proving some unique CFPtheorems under the generalized rational contraction conditions in FCM spaces by using compatibility and weakcompatibility of four self-mappings. We prove our results by using the one self-map are continuous. Furthermore, we prove some results without the continuity of self-mappings with supportive examples. In addition, we present an application of two nonlinear integral equations (NIEs) for the existence of a common solution to support our main work. This paper is managed as follows: in Section 2, we present the basic preliminary concept. While in Section 3, we prove our main results for unique CFP-theorems under the generalized rational contraction conditions in FCM spaces by using compatibility and weakly-compatibility of four selfmappings. In Section 4, we present NIEs as an application to support our main work.

\section{Preliminaries}

In this section, we recall some basic definitions and lemmas.

Definition 1 (see [34]). An operation $*:[0,1]^{2} \longrightarrow[0,1]$ is called a continuous $t$-norm if:

(i) $*$ is associative, commutative, and continuous

(ii) $1 * q_{1}=q_{1}$ and $q_{1} * q_{2} \leq q_{3} * q_{4}$, whenever $q_{1} \leq q_{3}$ and $q_{2} \leq q_{4}$, for all $q_{1}, q_{2}, q_{3}, q_{4} \in[0,1]$

Schweizer are Sklar [34] define the following basic continuous $t$-norms are

(i) The minimum; $q_{1} * q_{2}=\min \left\{q_{1}, q_{2}\right\}$

(ii) The product; $q_{1} * q_{2}=q_{1} q_{2}$

(iii) The Lukasiewicz; $q_{1} * q_{2}=\max \left\{q_{1}+q_{2}-1,0\right\}$

For detail study (see [34]).

Definition 2 (see [29]). A 3-tuple $\left(U, M_{o}, *\right)$ is called a FCM space if $C$ is a cone of $\mathbb{E}, U$ is an arbitrary set, $*$ is a contin- uous $t$-norm and $M_{o}$ is a fuzzy set on $U^{2} \times \operatorname{int}(P)$ satisfying the following conditions:

(1) $M_{o}\left(\lambda_{1}, \lambda_{2}, t\right)>0$ and $M_{o}\left(\lambda_{1}, \lambda_{2}, t\right)=1 \Leftrightarrow \lambda_{1}=\lambda_{2}$

(2) $M_{o}\left(\lambda_{1}, \lambda_{2}, t\right)=M_{o}\left(\lambda_{2}, \lambda_{1}, t\right)$

(3) $M_{o}\left(\lambda_{1}, \lambda_{2}, t\right) * M_{o}\left(\lambda_{2}, \lambda_{3}, s\right) \leq M_{o}\left(\lambda_{1}, \lambda_{3}, t+s\right)$

(4) $M_{o}\left(\lambda_{1}, \lambda_{2},.\right)$ : int $(P) \longrightarrow[0,1]$ is continuous

$\forall \lambda_{1}, \lambda_{2}, \lambda_{3} \in U$ and $t, s \in \operatorname{int}(P)$.

Definition 3 (see [29]). Let $\left(U, M_{o}, *\right)$ be a FCM space, $\exists \lambda_{1}$ $\in U$ and $\left\{\lambda_{j}\right\}$ be any sequence in $U$.

(i) $\left\{\lambda_{j}\right\}$ converges to $\lambda_{1}$ if for any $c \in(0,1), t \gg \theta$, and $\exists j_{1} \in N$ such that $M_{o}\left(\lambda_{j}, \lambda_{1}, t\right)>1-c$, for $j \geq j_{1}$. This can be written as $\lim _{j \longrightarrow \infty} \lambda_{j}=\lambda_{1}$, or $\lambda_{j} \longrightarrow \lambda_{1}$ as $j \longrightarrow \infty$

(ii) $\left(\lambda_{j}\right)$ is Cauchy if for any $c \in(0,1), t \gg \theta$, and $\exists j_{1}$ $\in N$ such that $M_{o}\left(\lambda_{j}, \lambda_{k}, t\right)>1-c$, for $j, k \geq j_{1}$

(iii) $\left(U, M_{o}, *\right)$ is complete if every Cauchy sequence is convergent in $U$

(iv) $\left\{\lambda_{j}\right\}$ is FC contractive if $\exists a \in(0,1)$ so that

$\frac{1}{M_{o}\left(\lambda_{j}, \lambda_{j+1}, t\right)}-1 \leq a\left(\frac{1}{M_{o}\left(\lambda_{j-1}, \lambda_{j}, t\right)}-1\right)$, for $t \gg \theta, j \geq 1$.

Lemma 4 (see [29]). "Let $\left(U, M_{o}, *\right)$ be a FCM space and a sequence $\lambda_{j} \longrightarrow \lambda_{1} \in U$ iff $M_{o}\left(\lambda_{j}, \lambda_{1}, t\right) \longrightarrow 1$ as $j \longrightarrow \infty$ for each $t \gg \theta^{\prime \prime}$.

Definition 5 (see [30]). Let $\left(U, M_{o}, *\right)$ be a FCM space. The FCM $M_{o}$ is triangular if

$$
\begin{aligned}
\frac{1}{M_{o}\left(\lambda_{1}, \lambda_{3}, t\right)}-1 \leq & \left(\frac{1}{M_{o}\left(\lambda_{1}, \lambda_{2}, t\right)}-1\right) \\
& +\left(\frac{1}{M_{o}\left(\lambda_{2}, \lambda_{3}, t\right)}-1\right), \forall \lambda_{1}, \lambda_{2}, \lambda_{3} \in U, t \gg \theta .
\end{aligned}
$$

Definition 6 (see [29]). Let $\left(U, M_{o}, *\right)$ be a FCM space and $A: U \longrightarrow U$. Then, $A$ is said to be FC contractive if there is $a \in(0,1)$ so that

$$
\frac{1}{M_{o}\left(A \lambda_{1}, A \lambda_{2}, t\right)}-1 \leq a\left(\frac{1}{M_{o}\left(\lambda_{1}, \lambda_{2}, t\right)}-1\right),
$$

$$
\forall \lambda_{1}, \lambda_{2} \in U \text {, and } t \gg \theta \text {. }
$$

Definition 7 (see [23]). Let $U \neq \varnothing$ set and let $B, h: U \longrightarrow U$ be the self-mappings on $U$. If there exists $\xi \in U$ such that $B$ $\rho=h \rho=\xi$ for some $\rho \in U$. Then, $\rho$ is called a coincidence 
point of $B$ and $h$, and $\xi$ is known as a point of coincidence of the mappings $B, h$. A pair of self-mappings $(B, h)$ is known to be weakly-compatible if the self-mappings commute at their coincidence point, i.e., $h B(\rho)=B h(\rho)$ for $\rho \in U$.

Proposition 8 (see [23]). Let $B, h$ be weakly-compatible selfmappings on $U$. If $B$ and $h$ have a unique point of coincidence, that is, $B \rho=h \rho=\xi$, then, $\xi$ is a unique CFP of the mappings $B$ and $h$.

Definition 9 (see [32]). A self-mapping pair $(h, B)$ is said to be compatible on a FCM space $\left(U, M_{o}, *\right)$, if, $\lim _{j \rightarrow \infty} M_{o}(B h$ $\left.\lambda_{j}, h B \lambda_{j}, t\right)=1$ for $t \gg \theta$, whenever $\left\{\lambda_{j}\right\}$ is a sequence in $U$ so that $\lim _{j \longrightarrow \infty} B \lambda_{j}=\lim _{j \longrightarrow \infty} h \lambda_{j}=\xi$ for some $\xi \in U$.

\section{Main Results}

Now, we are in the position to present our main results.

Theorem 10. Let $A, B, g, h: U \longrightarrow U$ be the four selfmappings on a complete FCM space $\left(U, M_{o}\right.$,*) in which a FCM $M_{o}$ is triangular and satisfies

$$
\begin{aligned}
\frac{1}{M_{o}(A \lambda, B \mu, t)}-1 \leq & a\left(\frac{1}{M_{o}(h \lambda, g \mu, t)}-1\right) \\
& +b\left(\frac{M_{o}(h \lambda, g \mu, t)}{M_{o}(h \lambda, B \mu, 2 t) * M_{o}(g \mu, A \lambda, 2 t)}-1\right) \\
& +c\left(\frac{1}{M_{r}(h \lambda, A \lambda, t)}-1+\frac{1}{M_{r}(g \mu, B \mu, t)}-1\right) \\
& +d\left(\frac{1}{M_{r}(g \mu, A \lambda, t)}-1+\frac{1}{M_{r}(h \lambda, B \mu, t)}-1\right),
\end{aligned}
$$

$\forall \lambda, \mu \in U, t \gg \theta$, and $0 \leq a, b, c, d<1$ with $(a+b+2 c+$ $2 d)<1$. If $A(U) \subseteq g(U), B(U) \subseteq h(U)$ and consider that [(1)]

(1) $h$ is a continuous self-mapping

(2) A pair $(A, h)$ is compatible, and

(3) A pair $(B, g)$ is weakly-compatible $U$.

Then, the mappings $A, B, g$, and $h$ have a unique CFP in

Proof. Fix $\lambda_{0} \in U$ and by the hypothesis $A(U) \subseteq g(U), B(U$ )$\subseteq h(U)$, we define the iterative sequences in $U$ so that

$$
\xi_{2 j+1}=g \lambda_{2 j+1}=A \lambda_{2 j} \text { and } \xi_{2 j+2}=h \lambda_{2 j+2}=B \lambda_{2 j+1}, j \geq 0 \text {. }
$$

Then, by (4),

$$
\begin{aligned}
& \frac{1}{M_{o}\left(g \lambda_{2 j+1}, h \lambda_{2 j+2}, t\right)}-1=\frac{1}{M_{o}\left(A \lambda_{2 j}, B \lambda_{2 j+1}, t\right)}-1 \\
& \leq a\left(\frac{1}{M_{o}\left(h \lambda_{2 j}, g \lambda_{2 j+1}, t\right)}-1\right) \\
& +b\left(\frac{M_{o}\left(h \lambda_{2 j}, g \lambda_{2 j+1}, t\right)}{M_{o}\left(h \lambda_{2 j}, B \lambda_{2 j+1}, 2 t\right) * M_{o}\left(g \lambda_{2 j+1}, A \lambda_{2 j}, 2 t\right)}-1\right) \\
& +c\left(\frac{1}{M_{o}\left(h \lambda_{2 j}, A \lambda_{2 j}, t\right)}-1+\frac{1}{M_{o}\left(g \lambda_{2 j+1}, B \lambda_{2 j+1}, t\right)}-1\right) \\
& +d\left(\frac{1}{M_{o}\left(g \lambda_{2 j+1}, A \lambda_{2 j}, t\right)}-1+\frac{1}{M_{o}\left(h \lambda_{2 j}, B \lambda_{2 j+1}, t\right)}-1\right) \\
& =a\left(\frac{1}{M_{o}\left(h \lambda_{2 j}, g \lambda_{2 j+1}, t\right)}-1\right)+b\left(\frac{M_{o}\left(h \lambda_{2 j}, g \lambda_{2 j+1}, t\right)}{M_{o}\left(h \lambda_{2 j}, h \lambda_{2 j+2}, 2 t\right)}-1\right) \\
& +c\left(\frac{1}{M_{o}\left(h \lambda_{2 j}, g \lambda_{2 j+1}, t\right)}-1+\frac{1}{M_{o}\left(g \lambda_{2 j+1}, h \lambda_{2 j+2}, t\right)}-1\right) \\
& +d\left(\frac{1}{M_{o}\left(h \lambda_{2 j}, h \lambda_{2 j+2}, t\right)}-1\right) \text {. }
\end{aligned}
$$

By Definition 2 (3), $M_{o}\left(h \lambda_{2 j}, h \lambda_{2 j+2}, 2 t\right) \geq M_{o}\left(h \lambda_{2 j}, g\right.$ $\left.\lambda_{2 j+1}, t\right) * M_{o}\left(g \lambda_{2 j+1}, h \lambda_{2 j+2}, t\right)$, for $t \gg \theta$. One writes

$$
\begin{aligned}
& \frac{1}{M_{o}\left(g \lambda_{2 j+1}, h \lambda_{2 j+2}, t\right)}-1 \leq a\left(\frac{1}{M_{o}\left(h \lambda_{2 j}, g \lambda_{2 j+1}, t\right)}-1\right) \\
& \quad+b\left(\frac{M_{o}\left(h \lambda_{2 j}, g \lambda_{2 j+1}, t\right)}{M_{o}\left(h \lambda_{2 j}, g \lambda_{2 j+1}, t\right) * M_{r}\left(g \lambda_{2 j+1}, h \lambda_{2 j+2}, t\right)}-1\right) \\
& \quad+c\left(\frac{1}{M_{o}\left(h \lambda_{2 j}, g \lambda_{2 j+1}, t\right)}-1+\frac{1}{M_{o}\left(g \lambda_{2 j+1}, h \lambda_{2 j+2}, t\right)}-1\right) \\
& +d\left(\frac{1}{M_{o}\left(h \lambda_{2 j}, g \lambda_{2 j+1}, t\right)}-1+\frac{1}{M_{o}\left(g \lambda_{2 j+1}, h \lambda_{2 j+2}, t\right)}-1\right) .
\end{aligned}
$$

This implies that

$$
\frac{1}{M_{o}\left(g \lambda_{2 j+1}, h \lambda_{2 j+2}, t\right)}-1 \leq \beta\left(\frac{1}{M_{o}\left(h \lambda_{2 j}, g \lambda_{2 j+1}, t\right)}-1\right) \text {, for } t \gg \theta \text {, }
$$

where $\gamma=a+c+d / 1-b-c-d<1$ since $(a+b+2 c+2$ 
d) $<1$. Similarly,

$$
\begin{aligned}
& \frac{1}{M_{o}\left(h \lambda_{2 j+2}, g \lambda_{2 j+3}, t\right)}-1=\frac{1}{M_{o}\left(A \lambda_{2 j+2}, B \lambda_{2 j+1}, t\right)}-1 \\
& \leq a\left(\frac{1}{M_{o}\left(g \lambda_{2 j+1}, h \lambda_{2 j+2}, t\right)}-1\right) \\
& +b\left(\frac{M_{o}\left(g \lambda_{2 j+1}, h \lambda_{2 j+2}, t\right)}{M_{o}\left(h \lambda_{2 j+2}, B \lambda_{2 j+1}, 2 t\right) * M\left(g \lambda_{2 j+1}, A \lambda_{2 j+2}, 2 t\right)}-1\right) \\
& +c\left(\frac{1}{M_{o}\left(h \lambda_{2 j+2}, A \lambda_{2 j+2}, t\right)}-1+\frac{1}{M_{o}\left(g \lambda_{2 j+1}, B \lambda_{2 j+1}, t\right)}-1\right) \\
& +d\left(\frac{1}{M_{o}\left(g \lambda_{2 j+1}, A \lambda_{2 j+2}, t\right)}-1+\frac{1}{M_{o}\left(h \lambda_{2 j+2}, B \lambda_{2 j+1}, t\right)}-1\right) \\
& =a\left(\frac{1}{M_{o}\left(g \lambda_{2 j+1}, h \lambda_{2 j+2}, t\right)}-1\right)+b\left(\frac{M_{o}\left(g \lambda_{2 j+1}, h \lambda_{2 j+2}, t\right)}{M\left(g \lambda_{2 j+1}, g \lambda_{2 j+3}, 2 t\right)}-1\right) \\
& +c\left(\frac{1}{M_{o}\left(h \lambda_{2 j+2}, g \lambda_{2 j+3}, t\right)}-1+\frac{1}{M_{o}\left(g \lambda_{2 j+1}, h \lambda_{2 j+2}, t\right)}-1\right) \\
& +d\left(\frac{1}{M_{o}\left(g \lambda_{2 j+1}, g \lambda_{2 j+3}, t\right)}-1\right) .
\end{aligned}
$$

Again, by Definition 2 (3), $M_{o}\left(g \lambda_{2 j+1}, g \lambda_{2 j+3}, 2 t\right) \geq M_{o}($ $\left.g \lambda_{2 j+1}, h \lambda_{2 j+2}, t\right) * M_{o}\left(h \lambda_{2 j+2}, g \lambda_{2 j+3}, t\right)$, for $t \gg \theta$. We have

$$
\begin{aligned}
& \frac{1}{M_{o}\left(h \lambda_{2 j+2}, g \lambda_{2 j+3}, t\right)}-1 \leq a\left(\frac{1}{M_{o}\left(g \lambda_{2 j+1}, h \lambda_{2 j+2}, t\right)}-1\right) \\
& +b\left(\frac{M_{o}\left(g \lambda_{2 j+1}, h \lambda_{2 j+2}, t\right)}{M_{o}\left(g \lambda_{2 j+1}, h \lambda_{2 j+2}, t\right) * M_{r}\left(h \lambda_{2 j+2}, g \lambda_{2 j+3}, t\right)}-1\right) \\
& +c\left(\frac{1}{M_{o}\left(g \lambda_{2 j+1}, h \lambda_{2 j+2}, t\right)}-1+\frac{1}{M_{o}\left(h \lambda_{2 j+2}, g \lambda_{2 j+3}, t\right)}-1\right) \\
& +d\left(\frac{1}{M_{o}\left(g \lambda_{2 j+1}, h \lambda_{2 j+2}, t\right)}-1+\frac{1}{M_{o}\left(h \lambda_{2 j+2}, g \lambda_{2 j+3}, t\right)}-1\right) .
\end{aligned}
$$

This implies that

$$
\frac{1}{M_{o}\left(h \lambda_{2 j+2}, g \lambda_{2 j+3}, t\right)}-1 \leq \beta\left(\frac{1}{M_{o}\left(g \lambda_{2 j+1}, h \lambda_{2 j+2}, t\right)}-1\right) \text {, for } t \gg \theta,
$$

where the value of $\beta$ is the same as in (8). Now, from (3), (8), (11), and by induction, we have

$$
\begin{aligned}
\frac{1}{M_{o}\left(\xi_{2 j+2}, \xi_{2 j+3}, t\right)}-1 & \leq \beta\left(\frac{1}{M_{o}\left(\xi_{2 j+1}, \xi_{2 j+2}, t\right)}-1\right) \\
& \leq \beta^{2}\left(\frac{1}{M_{o}\left(\xi_{2 j+1}, \xi_{2 j+2}, t\right)}-1\right) \\
& \leq \cdots \leq \beta^{2 j+2}\left(\frac{1}{M_{o}\left(\xi_{0}, \xi_{1}, t\right)}-1\right) \longrightarrow 0 \text {, as } j \longrightarrow \infty .
\end{aligned}
$$

Its prove that a sequence $\left\{\xi_{j}\right\}_{j \geq 0}$ is a FC contractive, and we get that

$$
\lim _{j \longrightarrow \infty} M_{o}\left(\xi_{j}, \xi_{j+1}, t\right)=1 \text {, for } t \gg \theta .
$$

Since $M_{o}$ is triangular, then $\forall k>j \geq j_{0}$,

$$
\begin{aligned}
\frac{1}{M_{o}\left(\xi_{j}, \xi_{k}, t\right)}-1 \leq & \left(\frac{1}{M_{o}\left(\xi_{j}, \xi_{j+1}, t\right)}-1\right)+\left(\frac{1}{M_{o}\left(\xi_{j+1}, \xi_{j+2}, t\right)}-1\right) \\
& +\cdots+\left(\frac{1}{M_{o}\left(\xi_{k-1}, \xi_{k}, t\right)}-1\right) \\
\leq & \left(\beta^{j}+\beta^{j+1}+\cdots+\beta^{k-1}\right)\left(\frac{1}{M_{o}\left(\xi_{0}, \xi_{1}, t\right)}-1\right) \\
\leq & \frac{\beta^{j}}{1-\beta}\left(\frac{1}{M_{o}\left(\xi_{0}, \xi_{1}, t\right)}-1\right) \longrightarrow 0, \text { as } j \longrightarrow \infty .
\end{aligned}
$$

Hence, proved that $\left\{\xi_{j}\right\}$ is a Cauchy sequence. Now by the completeness of FCM space $\left(U, M_{o}, *\right), \exists \xi \in U$ so that $\xi_{j} \longrightarrow \xi$ as $j \longrightarrow \infty$. Now for its subsequences, we have that

$g \lambda_{2 j+1} \longrightarrow \xi, h \lambda_{2 j+2} \longrightarrow \xi, A \lambda_{2 j} \longrightarrow \xi$, and $B \lambda_{2 j+1} \longrightarrow \xi$ as $j \longrightarrow \infty$.

Since, a self-mapping $h: U \longrightarrow U$ is continuous, therefore

$h\left(g \lambda_{2 j+1}\right) \longrightarrow h \xi, h\left(h \lambda_{2 j+2}\right) \longrightarrow h \xi, h\left(A \lambda_{2 j}\right) \longrightarrow h \xi$, and $h\left(B \lambda_{2 j+1}\right) \longrightarrow h \xi$ as $j \longrightarrow \infty$.

By hypothesis $(2)$, a $(A, h)$ is compatible, therefore,

$$
\begin{aligned}
& \lim _{j \longrightarrow \infty} M_{o}\left(A\left(h \lambda_{2 j}\right), h\left(A \lambda_{2 j}\right), t\right)=\lim _{j \longrightarrow \infty} M_{r}\left(A\left(h \lambda_{2 j}\right), h \xi, t\right) \\
& \quad=1, \Rightarrow \lim _{j \longrightarrow \infty} M_{r}\left(h\left(A \lambda_{2 j}\right), h \xi, t\right)=1, \text { for } t \gg \theta .
\end{aligned}
$$
(3),

Next, we have to prove that $h \xi=\xi$, then, by Definition 2

$$
M_{o}(h \xi, \xi, 2 t) \geq M_{r}\left(h \xi, A\left(h \lambda_{2 j}, t\right) * M_{r}\left(A\left(h \lambda_{2 j}, \xi, t\right) \text { for } t \gg \theta .\right.\right.
$$

Since, a pair $(A, h)$ is compatible, by using limit $j \longrightarrow \infty$, and by the view of (15), (17), and (18), we have

$$
\begin{aligned}
M_{o}(h \xi, \xi, 2 t) & \geq \lim _{j \longrightarrow \infty} M_{r}\left(h \xi, A\left(h \lambda_{2 j}, t\right) * \lim _{j \longrightarrow \infty} M_{r}\left(A\left(h \lambda_{2 j}, \xi, t\right)\right.\right. \\
& =1 * 1=1 \text { for } t \gg \theta .
\end{aligned}
$$

Hence, $M_{o}(h \xi, \xi, 2 t)=1 \Rightarrow h \xi=\xi$, for $t \gg \theta$. Now, we 
prove that $A \xi=\xi$, then again by Definition 2 (3),

$$
M_{o}(A \xi, \xi, 2 t) \geq M_{r}\left(A \xi, h\left(A \lambda_{2 j}, t\right) * M_{r}\left(h\left(A \lambda_{2 j}, \xi, t\right) \text { for } t \gg \theta\right. \text {. }\right.
$$

Again by using limit $j \longrightarrow \infty$, and by the view of (15), (17), and (20), we have

$$
\begin{aligned}
M_{o}(A \xi, \xi, 2 t) & \geq \lim _{j \longrightarrow \infty} M_{r}\left(A \xi, h\left(A \lambda_{2 j}\right), t\right) * \lim _{j \longrightarrow \infty} M_{r}\left(h\left(A \lambda_{2 j}\right), \xi, t\right) \\
& =1 * 1=1 \text { for } t \gg \theta .
\end{aligned}
$$

Hence, $M_{o}(A \xi, \xi, 2 t)=1 \Rightarrow A \xi=\xi$, for $t \gg \theta$. Thus, we get that $A \xi=h \xi=\xi$. Next, we have to prove that $B \xi=g \xi$. Now by hypothesis (1), i.e., $A(U) \subseteq g(U)$, and there exists $\rho \in U$ such that $\xi=A \xi=g \rho$. Then, by view of (4), for $t \gg \theta$,

$$
\begin{aligned}
\frac{1}{M_{o}(B \rho, g \rho, t)}-1= & \frac{1}{M_{o}(A \xi, B \rho, t)}-1 \leq a\left(\frac{1}{M_{o}(h \xi, g \rho, t)}-1\right) \\
& +b\left(\frac{M_{o}(h \xi, g \rho, t)}{M_{o}(h \xi, B \rho, 2 t) * M_{o}(g \rho, A \xi, 2 t)}-1\right) \\
& +c\left(\frac{1}{M_{o}(h \xi, A \xi, t)}-1+\frac{1}{M_{o}(g \rho, B \rho, t)}-1\right) \\
& +d\left(\frac{1}{M_{o}(g \rho, A \xi, t)}-1+\frac{1}{M_{o}(h \xi, B \rho, t)}-1\right) \\
= & a\left(\frac{1}{M_{o}(h \xi, \xi, t)}-1\right)+b\left(\frac{M_{o}(h \xi, g \rho, t)}{M_{o}(h \xi, B \rho, 2 t) * M_{o}(\xi, A \xi, 2 t)}-1\right) \\
& +c\left(\frac{1}{M_{o}(h \xi, \xi, t)}-1+\frac{1}{M_{o}(g \rho, B \rho, t)}-1\right) \\
& +d\left(\frac{1}{M_{o}(\xi, A \xi, t)}-1+\frac{1}{M_{o}(h \xi, B \rho, t)}-1\right) \\
= & b\left(\frac{M_{o}(h \xi, g \rho, t)}{M_{o}(h \xi, B \rho, 2 t)}-1\right)+c\left(\frac{1}{M_{o}(g \rho, B \rho, t)}-1\right) \\
& +d\left(\frac{1}{M_{o}(g \rho, B \rho, t)}-1\right) .
\end{aligned}
$$

Again, by Definition 2 (3), $M_{o}(h \xi, B \rho, 2 t) \geq M_{o}(h \xi, g \rho$, $t) * M_{o}(g \rho, B \rho, t)$, for $t \gg \theta$. It follows that

$$
\begin{aligned}
\frac{1}{M_{o}(B \rho, g \rho, t)}-1 \leq & b\left(\frac{M_{o}(h \xi, g \rho, t)}{M_{o}(h \xi, g \rho, t) * M_{o}(g \rho, B \rho, t)}-1\right) \\
& +(c+d)\left(\frac{1}{M_{o}(g \rho, B \rho, t)}-1\right) \\
= & (b+c+d)\left(\frac{1}{M_{o}(g \rho, B \rho, t)}-1\right) \text { for } t \gg \theta
\end{aligned}
$$

Noticing that $(b+c+d)<1$, therefore, $M_{o}(B \rho, g \rho, t)=$ $1 \Rightarrow B \rho=g \rho$ for $t \gg \theta$, hence, $B \rho=g \rho=\xi$. Now by hypothesis (3), a pair $(B, g)$ is weakly compatible, therefore,

$$
g \xi=g(B \rho)=B(g \rho)=B \xi
$$

Next, we have to prove that $B \xi=\xi$, then again by view of
(4) and by using Definition 2 (3), for $t \gg \theta$,

$$
\begin{aligned}
& \frac{1}{\mathrm{M}_{o}(B \xi, \xi, t)}-1=\frac{1}{M_{o}(B \xi, A \xi, t)}-1 \leq a\left(\frac{1}{M_{o}(h \xi, g \xi, t)}-1\right) \\
& \quad+b\left(\frac{M_{o}(h \xi, g \xi, t)}{M_{o}(h \xi, B \xi, 2 t) * M_{o}(g \xi, A \xi, 2 t)}-1\right) \\
& \quad+c\left(\frac{1}{M_{r}(h \xi, A \xi, t)}-1+\frac{1}{M_{r}(g \xi, B \xi, t)}-1\right) \\
& \quad+d\left(\frac{1}{M_{r}(g \xi, A \xi, t)}-1+\frac{1}{M_{r}(h \xi, B \xi, t)}-1\right) \\
& \quad a\left(\frac{1}{M_{o}(\xi, B \xi, t)}-1\right) \\
& \quad+b\left(\frac{M_{o}(h \xi, g \xi, t)}{M_{o}(h \xi, g \xi, t) * M_{o}(g \xi, B \xi, t) * M_{o}(g \xi, \xi, t) * M_{o}(\xi, A \xi, t)}-1\right) \\
& \quad+c\left(\frac{1}{M_{r}(h \xi, \xi, t)}-1+\frac{1}{M_{r}(g \xi, B \xi, t)}-1\right) \\
& \quad+d\left(\frac{1}{M_{r}(B \xi, \xi, t)}-1+\frac{1}{M_{r}(\xi, B \xi, t)}-1\right) .
\end{aligned}
$$

After simplification, we obtain

$$
\begin{aligned}
\frac{1}{M_{r}(B \xi, \xi, t)}-1 & \leq(a+b+2 d)\left(\frac{1}{M_{r}(B \xi, \xi, t)}-1\right), \\
& \Rightarrow(1-a-b-2 d)\left(\frac{1}{M_{r}(B \xi, \xi, t)}-1\right) \leq 0 \text {, for } t \gg \theta .
\end{aligned}
$$

Since $(1-a-b-2 d) \neq 0$, therefore, $M_{r}(B \xi, \xi, t)=1 \Rightarrow$ $B \xi=\xi$, for $t \gg \theta$, which further implies that $g \xi=\xi$. Hence, proved that $h \xi=g \xi=A \xi=B \xi=\xi$, that is, $\xi$ is the CFP of the mappings $A, B, g$, and $h$.

Uniqueness: let $\eta \in U$ be the other CFP of the mappings $A, B, g$, and $h$ in $U$ such that $h \eta=g \eta=A \eta=B \eta=\eta$. Then by view of (4) and by using Definition 2 (3), for $t \gg \theta$,

$$
\begin{aligned}
\frac{1}{M_{r}(\xi, \eta, t)}-1= & \frac{1}{M_{r}(A \xi, B \eta, t)}-1 \leq a\left(\frac{1}{M_{o}(h \xi, g \eta, t)}-1\right) \\
& +b\left(\frac{M_{o}(h \xi, g \eta, t)}{M_{o}(h \xi, B \eta, 2 t) * M_{o}(g \eta, A \xi, 2 t)}-1\right) \\
& +c\left(\frac{1}{M_{r}(h \xi, A \xi, t)}-1+\frac{1}{M_{r}(g \eta, B \eta, t)}-1\right) \\
& +d\left(\frac{1}{M_{r}(g \eta, A \xi, t)}-1+\frac{1}{M_{r}(h \xi, B \eta, t)}-1\right) \\
\leq & a\left(\frac{1}{M_{o}(\xi, \eta, t)}-1\right) \\
& +b\left(\frac{M_{o}(\xi, \xi, t) * M_{o}(\xi, \eta, t) * M_{o}(\eta, \eta, t) * M_{o}(\eta, \xi, t)}{M_{o}}-1\right) \\
& +2 d\left(\frac{1}{M_{r}(\eta, \xi, t)}-1\right) .
\end{aligned}
$$


After simplification, we obtain

$$
\begin{aligned}
\frac{1}{M_{r}(\xi, \eta, t)}-1 & \leq(a+b+2 d)\left(\frac{1}{M_{r}(\xi, \eta, t)}-1\right), \\
& \Rightarrow(1-a-b-2 d)\left(\frac{1}{M_{r}(\xi, \eta, t)}-1\right) \leq 0 \text {, for } t \gg \theta .
\end{aligned}
$$

Since $(1-a-b-2 d) \neq 0$, therefore, $M_{r}(\xi, \eta, t)=1 \Rightarrow \xi$ $=\eta$, for $t \gg \theta$. This completes the proof.

Corollary 11. Let $A, B, g, h: U \longrightarrow U$ be the four selfmappings on a complete FCM space $\left(U, M_{o}\right.$,*) in which a FCM $M_{o}$ is triangular and satisfies

$$
\begin{aligned}
\frac{1}{M_{o}(A \lambda, B \mu, t)}-1 \leq & a\left(\frac{1}{M_{o}(h \lambda, g \mu, t)}-1\right) \\
& +b\left(\frac{M_{o}(h \lambda, g \mu, t)}{M_{o}(h \lambda, B \mu, 2 t) * M_{o}(g \mu, A \lambda, 2 t)}-1\right) \\
& +c\left(\frac{1}{M_{r}(h \lambda, A \lambda, t)}-1+\frac{1}{M_{r}(g \mu, B \mu, t)}-1\right),
\end{aligned}
$$

$\forall \lambda, \mu \in U, t \gg \theta$, and $0 \leq a, b, c<1$ with $(a+b+2 c)<1$. If $A(U) \subseteq g(U), B(U) \subseteq h(U)$ and consider that

(1) $h$ is a continuous self-mapping

(2) A pair $(A, h)$ is compatible, and

(3) A pair $(B, g)$ is weakly-compatible $U$.

Then, the mappings $A, B, g$, and $h$ have a unique CFP in

Corollary 12. Let $A, B, g, h: U \longrightarrow U$ be the four selfmappings on a complete FCM space $\left(U, M_{o}\right.$,*) in which a FCM $M_{o}$ is triangular and satisfies

$$
\begin{aligned}
& \left.\frac{1}{M_{o}(} A \lambda, B \mu, t\right)-1 \leq a\left(\frac{1}{M_{o}(h \lambda, g \mu, t)}-1\right) \\
& \quad+b\left(\frac{M_{o}(h \lambda, g \mu, t)}{M_{o}(h \lambda, B \mu, 2 t) * M_{o}(g \mu, A \lambda, 2 t)}-1\right) \\
& \quad+d\left(\frac{1}{M_{r}(g \mu, A \lambda, t)}-1+\frac{1}{M_{r}(h \lambda, B \mu, t)}-1\right),
\end{aligned}
$$

$\forall \lambda, \mu \in U, t \gg \theta$, and $0 \leq a, b, d<1$ with $(a+b+2 d)<1$. If $A(U) \subseteq g(U), B(U) \subseteq h(U)$ and consider that

(1) $h$ is a continuous self-mapping

(2) A pair $(A, h)$ is compatible, and

(3) A pair $(B, g)$ is weakly-compatible $U$.

Then, the mappings $A, B, g$, and $h$ have a unique CFP in
Corollary 13. Let $A, B, g, h: U \longrightarrow U$ be the four selfmappings on a complete FCM space $\left(U, M_{o}, *\right)$ in which a FCM $M_{o}$ is triangular and satisfies

$$
\begin{aligned}
& \frac{1}{M_{o}(A \lambda, B \mu, t)}-1 \leq a\left(\frac{1}{M_{o}(h \lambda, g \mu, t)}-1\right) \\
& +c\left(\frac{1}{M_{r}(h \lambda, A \lambda, t)}-1+\frac{1}{M_{r}(g \mu, B \mu, t)}-1\right) \\
& \quad+d\left(\frac{1}{M_{r}(g \mu, A \lambda, t)}-1+\frac{1}{M_{r}(h \lambda, B \mu, t)}-1\right),
\end{aligned}
$$

$\forall \lambda, \mu \in U, t \gg \theta$, and $0 \leq a, c, d<1$ with $(a+2 c+2 d)<1$ . If $A(U) \subseteq g(U), B(U) \subseteq h(U)$ and consider that

(1) $h$ is a continuous self-mapping

(2) A pair $(A, h)$ is compatible, and

(3) A pair $(B, g)$ is weakly-compatible

Then, the mappings $A, B, g$, and $h$ have a CFP in $U$.

Example 14. Assume that $U=[0, \infty), *$ is a continuous $t$ -norm and $M_{0}: U \times U \times(0, \infty) \longrightarrow[0,1]$ be written as

$$
M_{o}(\lambda, \mu, t)=\frac{t}{t+|\lambda-\mu|}, \forall \lambda, \mu \in U, t \gg \theta
$$

Then, it is easy to verify that FCM $M_{o}$ is triangular and $\left(U, M_{o}, *\right)$ is a complete FCM space. Now, the mappings, $A$ , $g, h, B: U \longrightarrow U$, be defined by, (for all $\lambda \in U$ );

$$
A(\lambda)=B(\lambda)=\left(\begin{array}{ll}
\frac{1}{3}\left(\frac{3 \lambda}{4}+\frac{1}{8}\right), & \text { if } \lambda \neq 0, \\
0, & \text { if } \lambda=0
\end{array}\right.
$$

and

$$
h(\lambda)=g(\lambda)=\left(\begin{array}{ll}
\left(\frac{3 \lambda}{4}+\frac{1}{8}\right), & \text { if } \lambda \neq 0, \\
0, & \text { if } \lambda=0 .
\end{array}\right.
$$

Since, from the above equation, $A(U)=B(U)$ and $g(U$ )$=h(U)$, so that we conclude that $A(U) \subseteq g(U)$ or $B(U) \subseteq$ $h(U)$. Then,

$$
\frac{1}{M_{o}(h \lambda, g \mu, t)}-1=\frac{|h \lambda-g \mu|}{t}=\frac{3|\lambda-\mu|}{4 t}, \text { for } t \gg \theta
$$

and

$$
\frac{1}{M_{o}(A \lambda, \mathrm{B} \mu, t)}-1=\frac{|A(\lambda)-B(\mu)|}{t}=\frac{1}{3}\left(\frac{1}{M_{o}(h \lambda, g \mu, t)}-1\right) \text { for } t \gg \theta .
$$

Hence, the mappings $A, B, g$, and $h$ on $U$ satisfying the 
fuzzy cone-contraction condition in FCM space. Now, from Definition 2 (3), $M_{r}(h \lambda, B \mu, 2 t) \geq M_{r}(h \lambda, g \mu, t) * M_{r}(g \mu, B$ $\mu, t)$ and $M_{r}(g \mu, A \lambda, 2 t) \geq M_{r}(g \mu, h \lambda, t) * M_{r}(h \lambda, A \lambda, t)$, for $t \gg \theta$. Now, we calculate the following terms of (4), for $t \gg \theta$,

$$
\begin{aligned}
& \quad \frac{M_{o}(h \lambda, g \mu, t)}{M_{o}(h \lambda, B \mu, 2 t) * M_{r}(g \mu, A \lambda, 2 t)}-1 \\
& \quad \leq \frac{1}{M_{o}(h \lambda, g \mu, t) * M_{r}(h \lambda, A \lambda, t) * M_{r}(g \mu, B \mu, t)}-1 \\
& \quad=\frac{1}{(t / t+|h \lambda-g \mu|) \cdot(t / t+|h \lambda-A \lambda|) \cdot(t / t+|g \mu-B \mu|)}-1 \\
& \quad=\frac{(t+|h \lambda-g \mu|) \cdot(t+|h \lambda-A \lambda|) \cdot(t+|g \mu-B \mu|)}{t^{3}}-1 \\
& \quad=\frac{(t+3 / 4|\lambda-\mu|) \cdot[(t+1 / 12(6 \lambda+1)) \cdot(t+1 / 12(6 \mu+1))]}{t^{3}}-1 \\
& \quad=\frac{(t+3 / 4|\lambda-\mu|) \cdot\left[1 / 144(6 \lambda+1) \cdot(6 \mu+1)+(t / 6)(3 \lambda+3 \mu+1)+\left(3 t^{2} / 4\right)|\lambda-\mu|\right]}{t^{3}} \\
& \quad=\frac{(t+3 / 4|\lambda-\mu|) \cdot[1 / 144(6 \lambda+1) \cdot(6 \mu+1)+(t / 6)(3 \lambda+3 \mu+1)]}{t^{3}}+\frac{3}{4 t}|\lambda-\mu| .
\end{aligned}
$$

Next, we calculate

$$
\begin{aligned}
& \left(\frac{1}{M_{r}(h \lambda, A \lambda, t)}-1+\frac{1}{M_{r}(g \mu, B \mu, t)}-1\right) \\
& \quad=\frac{1}{t / t+|h \lambda-A \lambda|}-1+\frac{1}{t / t+|g \mu-B \mu|}-1 \\
& \quad=\frac{|h \lambda-A \lambda|}{t}+\frac{|g \mu-B \mu|}{t}=\frac{1}{6 t}(3 \lambda+3 \mu+1) \text { for } t \gg \theta .
\end{aligned}
$$

Similarly,

$$
\begin{aligned}
& \left(\frac{1}{M_{r}(g \mu, A \lambda, t)}-1+\frac{1}{M_{r}(h \lambda, B \mu, t)}-1\right) \\
& \quad=\frac{1}{t / t+|g \mu-A \lambda|}-1+\frac{1}{t / t+|h \lambda-B \mu|}-1 \\
& \quad=\frac{|g \mu-A \lambda|}{t}+\frac{|h \lambda-B \mu|}{t}=\frac{1}{6 t}(3 \lambda+3 \mu+1) \text { for } t \gg \theta .
\end{aligned}
$$

Thus, after routine calculation, all the conditions of Theorem 10 are satisfied with $a=1 / 3, b=2 / 7$, and $c=d=1 / 14$, and the mappings $\mathrm{A}, B, g$, and $h$ on $U$ have a unique CFP, i.e., $0 \in U$.

If we choose the self-mappings $B=A$ and $g=h$ in Theorem 10 , we obtain the following corollary.

Corollary 15. Let $B, h: U \longrightarrow U$ be two self-mappings on a complete FCM space $\left(U, M_{o}\right.$,*) in which a FCM $M_{o}$ is triangular and satisfies

$$
\begin{aligned}
\frac{1}{M_{o}(B \lambda, B \mu, t)}-1 \leq & a\left(\frac{1}{M_{o}(h \lambda, h \mu, t)}-1\right) \\
& +b\left(\frac{M_{o}(h \lambda, h \mu, t)}{M_{o}(h \lambda, B \mu, 2 t) * M_{o}(h \mu, B \lambda, 2 t)}-1\right) \\
& +c\left(\frac{1}{M_{r}(h \lambda, B \lambda, t)}-1+\frac{1}{M_{r}(h \mu, B \mu, t)}-1\right) \\
& +d\left(\frac{1}{M_{r}(h \mu, B \lambda, t)}-1+\frac{1}{M_{r}(h \lambda, B \mu, t)}-1\right),
\end{aligned}
$$

$\forall \lambda, \mu \in U, t \gg \theta$, and $0 \leq a, b, c, d<1$ with $(a+b+2 c+$ $2 d)<1$. If $B(U) \subseteq h(U), h$ is a continuous self-mapping, and a pair $(B, h)$ is weakly-compatible. Then, the mappings $B$ and $h$ have a unique CFP in $U$.

Next result, we shall prove without the continuity of selfmapping, i.e., $h$ and we replaced the completeness of $U$ by the completeness of $B(U)$ or $h(U)$.

Theorem 16. Let $B, h: U \longrightarrow U$ be two self-mappings on a complete FCM space $\left(U, M_{o}\right.$,*) in which a FCM $M_{o}$ is triangular and satisfies

$$
\begin{aligned}
\frac{1}{M_{o}(B \lambda, B \mu, t)}-1 \leq & a\left(\frac{1}{M_{o}(h \lambda, h \mu, t)}-1\right) \\
& +b\left(\frac{M_{o}(h \lambda, h \mu, t)}{M_{o}(h \lambda, B \mu, 2 t) * M_{o}(h \mu, B \lambda, 2 t)}-1\right) \\
& +c\left(\frac{1}{M_{r}(h \lambda, B \lambda, t)}-1+\frac{1}{M_{r}(h \mu, B \mu, t)}-1\right) \\
& +d\left(\frac{1}{M_{r}(h \mu, B \lambda, t)}-1+\frac{1}{M_{r}(h \lambda, B \mu, t)}-1\right),
\end{aligned}
$$

$\forall \lambda, \mu \in U, t \gg \theta$, and $0 \leq a, b, c, d<1$ with $(a+b+2 c+$ $2 d)<1$. If $B(U) \subseteq h(U), B(U)$ or $h(U)$ is complete and a pair $(B, h)$ is weakly-compatible. Then, the mappings $B$ and $h$ have a unique CFP in $U$.

Proof. From the proof of Theorem 10, we assume that $\left\{\xi_{j}\right\}_{j \geq 0}$ is a Cauchy sequence in $h(U)$, and the iterative sequences are earlier defined in the proof of Theorem 10, that are,

$$
\xi_{2 j+1}=h \lambda_{2 j+1}=B \lambda_{2 j} \text { and } \xi_{2 j+2}=h \lambda_{2 j+2}=B \lambda_{2 j+1}, j \geq 0
$$

We know that $h(U)$ is complete, and $\exists \xi, \rho \in U$, so that $\xi_{2 j+1} \longrightarrow \xi=h \rho$, as $j \longrightarrow \infty$. Therefore,

$\lim _{j \longrightarrow \infty} M_{o}\left(\xi_{2 i+1}, \xi, t\right)=\lim _{j \longrightarrow \infty} M_{o}\left(h \lambda_{2 j+1}, \xi, t\right)=1$ for $t \gg \theta$ 
Since by $M_{o}$ triangular property,

$\frac{1}{M_{o}(h \rho, B \rho, t)}-1 \leq\left(\frac{1}{M_{o}\left(h \rho, \xi_{2 j+2}, t\right)}-1\right)+\left(\frac{1}{M_{o}\left(\xi_{2 j+2}, B \rho, t\right)}-1\right)$ for $t \gg \theta$.

Now by the view of (41), (43), and by using Definition 2 (3), for $t \gg \theta$, we have that

$$
\begin{aligned}
& \frac{1}{M_{o}\left(\xi_{2 j+2}, B \rho, t\right)}-1=\frac{1}{M_{o}\left(B \lambda_{2 j+1}, B \rho, t\right)}-1 \leq a\left(\frac{1}{M_{o}\left(h \lambda_{2 j+1}, h \rho, t\right)}-1\right) \\
& \quad+b\left(\frac{M_{o}\left(h \lambda_{2 j+1}, h \rho, t\right)}{M_{o}\left(h \lambda_{2 j+1}, B \rho, 2 t\right) * M_{o}\left(h \rho, B \lambda_{2 j+1}, 2 t\right)}-1\right) \\
& \quad+c\left(\frac{1}{M_{r}\left(h \lambda_{2 j+1}, B \lambda_{2 j+1}, t\right)}-1+\frac{1}{M_{r}(h \rho, B \rho, t)}-1\right) \\
& \quad+d\left(\frac{1}{M_{r}\left(h \rho, B \lambda_{2 j+1}, t\right)}-1+\frac{1}{M_{r}\left(h \lambda_{2 j+1}, B \rho, t\right)}-1\right) \\
&=a\left(\frac{1}{M_{o}\left(h \lambda_{2 j+1}, h \rho, t\right)}-1\right)+b\left(\frac{1}{M_{o}\left(h \lambda_{2 j+1}, B \rho, 2 t\right) * M_{o}\left(h \rho, h \lambda_{2 j+2}, 2 t\right)}-1\right) \\
& \quad+c\left(\frac{1}{M_{r}\left(h \lambda_{2 j+1}, h \lambda_{2 j+2}, t\right)}-1+\frac{1}{M_{r}(h \rho, B \rho, t)}-1\right) \\
& \quad+d\left(\frac{1}{M_{r}\left(h \rho, h \lambda_{2 j+2}, t\right)}-1+\frac{1}{M_{r}\left(h \lambda_{2 j+1}, B \rho, t\right)}-1\right) \\
& \leq a\left(\frac{1}{M_{o}\left(h \lambda_{2 j+1}, h \rho, t\right)}-1\right) \\
& \quad+b\left(\frac{1}{M_{o}\left(h \lambda_{2 j+1}, h \rho, t\right) * M_{o}(h \rho, B \rho, t) * M_{o}\left(h \rho, h \lambda_{2 j+2}, 2 t\right)}-1\right) \\
&+c\left(\frac{1}{M_{r}\left(h \lambda_{2 j+1}, h \lambda_{2 j+2}, t\right)}-1+\frac{1}{M_{r}(h \rho, B \rho, t)}-1\right) \\
&+d\left(\frac{1}{M_{r}\left(h \rho, h \lambda_{2 j+2}, t\right)}-1+\frac{1}{M_{r}\left(h \lambda_{2 j+1}, B \rho, t\right)}-1\right) \\
& \longrightarrow(b+c+d)\left(\frac{1}{M_{r}(h \rho, B \rho, t)}-1\right), \mathrm{as} j \longrightarrow \infty .
\end{aligned}
$$

Hence,

$$
\limsup _{j \longrightarrow \infty}\left(\frac{1}{M_{o}\left(\xi_{2 j+2}, B \rho, t\right)}-1\right) \leq(b+c+d)\left(\frac{1}{M_{r}(h \rho, B \rho, t)}-1\right), \text { for } t \gg \theta .
$$

Now from (44) and (46),

$$
\begin{aligned}
\frac{1}{M_{o}(h \rho, B \rho, t)}-1 & \leq(b+c+d)\left(\frac{1}{M_{r}(h \rho, B \rho, t)}-1\right), \\
& \Rightarrow(1-b-c-d)\left(\frac{1}{M_{r}(h \rho, B \rho, t)}-1\right) \leq 0 \text {, for } t \gg \theta .
\end{aligned}
$$

Since, $(1-b-c-d) \neq 0$, therefore, $M_{r}(h \rho, B \rho, t)=1 \Rightarrow$ $h \rho=B \rho=\xi$, for $t \gg \theta$. Now by the weak-compatibility of ( $B, h)$,

$$
B \xi=B(h \rho)=h(B \rho)=h \xi .
$$

Next, we have to prove that $B \xi=\xi$. Then, again by view of (41) and by using Definition 2 (3), for $t \gg \theta$, we have

$$
\begin{aligned}
& \frac{1}{M_{o}(B \xi, \xi, t)}-1=\frac{1}{M_{o}(B \xi, B \rho, t)}-1 \leq a\left(\frac{1}{M_{o}(h \xi, h \rho, t)}-1\right) \\
& \quad+b\left(\frac{M_{o}(h \xi, h \rho, t)}{M_{o}(h \xi, B \rho, 2 t) * M_{o}(h \rho, B \xi, 2 t)}-1\right) \\
& \quad+c\left(\frac{1}{M_{r}(h \xi, B \xi, t)}-1+\frac{1}{M_{r}(h \rho, B \rho, t)}-1\right) \\
& \quad+d\left(\frac{1}{M_{r}(h \rho, B \xi, t)}-1+\frac{1}{M_{r}(h \xi, B \rho, t)}-1\right) \\
& \leq a\left(\frac{1}{M_{o}(B \xi, \xi, t)}-1\right) \\
& \quad+b\left(\frac{1}{M_{o}(h \xi, h \rho, t) * M_{o}(h \rho, B \rho, t) * M_{o}(h \rho, \xi, t) * M_{o}(\xi, B \xi, t)}-1\right) \\
& \quad+c\left(\frac{1}{M_{r}(h \xi, B \xi, t)}-1+\frac{1}{M_{r}(h \rho, B \rho, t)}-1\right) \\
& +d\left(\frac{1}{M_{r}(\xi, B \xi, t)}-1+\frac{1}{M_{r}(B \xi, \xi, t)}-1\right) \\
& =(a+b+2 d)\left(\frac{1}{M_{r}(\xi, B \xi, t)}-1\right) .
\end{aligned}
$$

Hence,

$$
\begin{aligned}
\frac{1}{M_{o}(B \xi, \xi, t)}-1 & \leq(a+b+2 d)\left(\frac{1}{M_{r}(B \xi, \xi, t)}-1\right), \\
& \Rightarrow(1-a-b-2 d)\left(\frac{1}{M_{r}(B \xi, \xi, t)}-1\right) \\
& \leq 0, \text { for } t \gg \theta .
\end{aligned}
$$

As $(1-a-b-2 d) \neq 0$, therefore, $M_{r}(B \xi, \xi, t)=1 \Rightarrow B \xi$ $=\xi$, for $t \gg \theta$. Hence, proved that $B \xi=h \xi=\xi$.

Uniqueness: let $\exists \eta \in U$ be the other CFP of the mapping $B$ and $h$ in $U$ such that $B \eta=h \eta=\eta$. Then by the view of (41) and by using Definition 2 (3),

$$
\begin{aligned}
& \frac{1}{M_{o}(\xi, \eta, t)}-1=\frac{1}{M_{o}(B \xi, B \eta, t)}-1 \leq a\left(\frac{1}{M_{o}(h \xi, h \eta, t)}-1\right) \\
& \quad+b\left(\frac{M_{o}(h \xi, h \eta, t)}{M_{o}(h \xi, B \eta, 2 t) * M_{o}(h \eta, B \xi, 2 t)}-1\right) \\
& \quad+c\left(\frac{1}{M_{r}(h \xi, B \xi, t)}-1+\frac{1}{M_{r}(h \eta, B \eta, t)}-1\right) \\
& \quad+d\left(\frac{1}{M_{r}(h \eta, B \xi, t)}-1+\frac{1}{M_{r}(h \xi, B \eta, t)}-1\right) \\
& \leq a\left(\frac{1}{M_{o}(\xi, \eta, t)}-1\right) \\
&+b\left(\frac{M_{o}(\xi, \eta, t)}{M_{o}(\xi, \eta, t) * M_{o}(\eta, \eta, t) * M_{o}(\eta, \xi, t) * M_{o}(\xi, \xi, t)}-1\right) \\
&+c\left(\frac{1}{M_{r}(\xi, \xi, t)}-1+\frac{1}{M_{r}(\eta, \eta, t)}-1\right) \\
&+d\left(\frac{1}{M_{r}(\eta, \xi, t)}-1+\frac{1}{M_{r}(\xi, \eta, t)}-1\right)=(a+b+2 d)\left(\frac{1}{M_{r}(\eta, \xi, t)}-1\right) .
\end{aligned}
$$


Hence,

$$
\begin{aligned}
\frac{1}{M_{r}(\eta, \xi, t)}-1 & \leq(a+b+2 d)\left(\frac{1}{M_{r}(\eta, \xi, t)}-1\right), \\
& \Rightarrow(1-a-b-2 d)\left(\frac{1}{M_{r}(\eta, \xi, t)}-1\right) \leq 0, \text { for } t \gg \theta .
\end{aligned}
$$

As $(1-a-b-2 d) \neq 0$, therefore, $M_{r}(\xi, \eta, t)=1 \Rightarrow \xi=\eta$, for $t \gg \theta$. Hence, proved that the mappings $B, h$ have a unique CFP in $U$.

Corollary 17. Let $B, h: U \longrightarrow U$ be two self-mappings on a complete FCM space $\left(U, M_{o}, *\right)$ in which a FCM $M_{o}$ is triangular and satisfies

$$
\begin{aligned}
& \frac{1}{M_{o}(B \lambda, B \mu, t)}-1 \leq a\left(\frac{1}{M_{o}(h \lambda, h \mu, t)}-1\right) \\
& \quad+b\left(\frac{M_{o}(h \lambda, h \mu, t)}{M_{o}(h \lambda, B \mu, 2 t) * M_{o}(h \mu, B \lambda, 2 t)}-1\right) \\
& \quad+c\left(\frac{1}{M_{r}(h \lambda, B \lambda, t)}-1+\frac{1}{M_{r}(h \mu, B \mu, t)}-1\right),
\end{aligned}
$$

$\forall \lambda, \mu \in U, t \gg \theta$, and $0 \leq a, b, c<1$ with $(a+b+2 c)<1$. If $B(U) \subseteq h(U), B(U)$ or $h(U)$ is complete and a pair $(B, h)$ is weakly-compatible. Then, the mappings $B, h$ have a unique CFP in $U$.

Corollary 18. Let $B, h: U \longrightarrow U$ be two self-mappings on a complete FCM space $\left(U, M_{o}, *\right)$ in which a FCM $M_{o}$ is triangular and satisfies

$$
\begin{aligned}
& \frac{1}{M_{o}(B \lambda, B \mu, t)}-1 \leq a\left(\frac{1}{M_{o}(h \lambda, h \mu, t)}-1\right) \\
& \quad+b\left(\frac{M_{o}(h \lambda, h \mu, t)}{M_{o}(h \lambda, B \mu, 2 t) * M_{o}(h \mu, B \lambda, 2 t)}-1\right) \\
& \quad+d\left(\frac{1}{M_{r}(h \mu, B \lambda, t)}-1+\frac{1}{M_{r}(h \lambda, B \mu, t)}-1\right),
\end{aligned}
$$

$\forall \lambda, \mu \in U, t \gg \theta$, and $0 \leq a, b, d<1$ with $(a+b+2 d)<1$. If $B(U) \subseteq h(U), B(U)$ or $h(U)$ is complete and a pair $(B, h)$ is weakly-compatible. Then, the mappings $B, h$ have a unique CFP in $U$.

Corollary 19. Let $B, h: U \longrightarrow U$ be two self-mappings on a complete FCM space $\left(U, M_{o}, *\right)$ in which a FCM $M_{o}$ is triangular and satisfies

$$
\begin{aligned}
& \frac{1}{M_{o}(B \lambda, B \mu, t)}-1 \leq a\left(\frac{1}{M_{o}(h \lambda, h \mu, t)}-1\right) \\
& \quad+b\left(\frac{M_{o}(h \lambda, h \mu, t)}{M_{o}(h \lambda, B \mu, 2 t) * M_{o}(h \mu, B \lambda, 2 t)}-1\right),
\end{aligned}
$$

for all $\lambda, \mu \in U, t \gg \theta$, and $0 \leq a, b<1$ with $(a+b)<1$. If $B(U) \subseteq h(U), B(U)$ or $h(U)$ is complete and a pair $(B, h)$ is weakly-compatible. Then, the mappings $B, h$ have a unique CFP in $U$.

Corollary 20. Let $B, h: U \longrightarrow U$ be two self-mappings on a complete FCM space $\left(U, M_{o}, *\right)$ in which a FCM $M_{o}$ is triangular and satisfies

$$
\begin{aligned}
& \frac{1}{M_{o}(B \lambda, B \mu, t)}-1 \leq a\left(\frac{1}{M_{o}(h \lambda, h \mu, t)}-1\right) \\
& \quad+c\left(\frac{1}{M_{r}(h \lambda, B \lambda, t)}-1+\frac{1}{M_{r}(h \mu, B \mu, t)}-1\right) \\
& \quad+d\left(\frac{1}{M_{r}(h \mu, B \lambda, t)}-1+\frac{1}{M_{r}(h \lambda, B \mu, t)}-1\right),
\end{aligned}
$$

$\forall \lambda, \mu \in U, t \gg \theta$, and $0 \leq a, c, d<1$ with $(a+2 c+2 d)<1$ . If $B(U) \subseteq h(U), B(U)$ or $h(U)$ is complete and a pair $(B, h)$ is weakly-compatible. Then, the mappings $B, h$ have a unique CFP in $U$.

Example 21. From Example 14, let us define $B, h: U \longrightarrow U$, for all $\lambda \in U$ such that

$$
B(\lambda)=\left(\begin{array}{ll}
\frac{1}{6}\left(\frac{3 \lambda}{4}+\frac{1}{4}\right), & \text { if } \lambda \neq 0, \\
0, & \text { if } \lambda=0,
\end{array}\right.
$$

and

$$
h(\lambda)=\left(\begin{array}{ll}
\left(\frac{3 \lambda}{4}+\frac{1}{4}\right), & \text { if } \lambda \neq 0, \\
0, & \text { if } \lambda=0 .
\end{array}\right.
$$

Hence, from the above equations, we have that $B(U) \subseteq$ $h(U)$. Then, for $t \gg \theta$, we have that

$$
\begin{aligned}
\frac{1}{M_{o}(B \lambda, B \mu, t)}-1= & \frac{|B \lambda-B \mu|}{t}=\frac{|\lambda-\mu|}{8 t} \leq \frac{12 \lambda+3 \mu+5}{36 t} \\
\leq & \frac{1}{8 t}|\lambda-\mu|+\frac{5}{72 t}(3 \lambda+3 \mu+2) \\
= & \frac{1}{6 t}\left|\frac{3 \lambda}{4}-\frac{3 \mu}{4}\right|+\frac{5}{144 t}(3 \lambda+3 \mu+2)+\frac{5}{144 t}(3 \lambda+3 \mu+2) \\
= & \frac{1}{6 t}\left|\frac{3 \lambda}{4}-\frac{3 \mu}{4}\right|+\frac{1}{6 t}\left(\frac{5}{24}(3 \lambda+3 \mu+2)\right)+\frac{1}{6 t}\left(\frac{5}{24}(3 \lambda+3 \mu+2)\right) \\
\leq & \frac{1}{6}\left(\frac{1}{M_{o}(h \lambda, h \mu, t)}-1\right)+\frac{1}{6}\left(\frac{5}{24 t}(3 \lambda+1)+\frac{5}{24 t}(3 \mu+1)\right) \\
& +\frac{1}{6}\left(\frac{1}{24 t}|18 \mu-3 \lambda+5|+\frac{1}{24 t}|18 \lambda-3 \mu+5|\right) \\
= & \frac{1}{6}\left(\frac{1}{M_{o}(h \lambda, h \mu, t)}-1\right)+\frac{1}{6}\left(\left|\frac{h \lambda-B \lambda \mid}{t}\right|+\left|\frac{h \mu-B \mu}{t}\right|\right) \\
& +\frac{1}{6}\left(\left|\frac{h \mu-B \lambda \mid}{t}\right|+\left|\frac{h \lambda-B \mu}{t}\right|\right)=\frac{1}{6}\left(\frac{1}{M_{o}(h \lambda, h \mu, t)}-1\right) \\
& +c\left(\frac{1}{M_{r}(h \lambda, B \lambda, t)}-1+\frac{1}{M_{r}(h \mu, B \mu, t)}-1\right) \\
& +\frac{1}{6}\left(\frac{1}{M_{r}(h \mu, B \lambda, t)}-1+\frac{1}{M_{r}(h \lambda, B \mu, t)}-1\right) .
\end{aligned}
$$

Thus, after simplification, we conclude that all the 
conditions of Corollary 20 are satisfied with $a=c=d=1 / 6$ and the mappings $B, h$ have a unique CFP, i.e., $0 \in U$.

\section{Supportive Application}

This section will deal with the nonlinear integral equations (NIEs) to support our main work. Let $U=C([0, \varrho], \mathbb{R})$ be the space of all $\mathbb{R}$ - valued continuous functions on $[0, \varrho]$, where $0<\mathrm{Q} \in \mathbb{R}$. The two NIEs are

$\lambda(\tau)=\int_{0}^{\rho} Q_{1}(\tau, v, \lambda(v)) d v$, and $\mu(\tau)=\int_{0}^{\rho} Q_{2}(\tau, v, \mu(v)) d v, \forall \lambda, \mu \in U$,

where $\tau \in[0, \varrho]$ and $Q_{1}, Q_{2}:[0, \varrho] \times[0, \varrho] \times \mathbb{R} \longrightarrow \mathbb{R}$, and a metric $d: U \times U \longrightarrow \mathbb{R}$ is defined by

$d(\lambda, \mu)=\sup _{\tau \in[0, \mathrm{e}]}|\lambda(\tau)-\mu(\tau)|=\|\lambda-\mu\|$, where $\lambda, \mu \in C([0, \mathrm{e}], \mathbb{R})=U$.

The operation $*$ is defined by $\rho_{1} * \rho_{2}=\rho_{1} \rho_{2}$ for all $\rho_{1}$, $\rho_{2} \in[0, \mathrm{e}]$. A FM $M_{o}: U \times U \times(0, \infty) \longrightarrow[0,1]$ is defined by

$$
M_{r}(\lambda, \mu, t)=\frac{t}{t+d(\lambda, \mu)}, \text { for } t>0, \forall \lambda, \mu \in U .
$$

Then, FM $M_{o}$ is triangular and $\left(U, M_{o}, *\right)$ is a complete FCM space.

Theorem 22. The two NIEs are

$$
\lambda(\tau)=\int_{0}^{\varrho} Q_{1}(\tau, v, x(v)) d v, \text { and } \mu(\tau)=\int_{0}^{\varrho} Q_{2}(\tau, v, \mu(v)) d v,
$$

where $\tau, v \in[0, \mathrm{\varrho}]$ and $\lambda, \mu \in U$. Assume that $Q_{1}, Q_{2}:[0$, $\mathrm{\varrho}] \times[0, \mathrm{\varrho}] \times \mathbb{R} \longrightarrow \mathbb{R}$ are so that $A_{\lambda}, B_{\mu} \in U$ for all $\lambda, \mu \in U$, where

$$
A_{\lambda}(\tau)=\int_{0}^{\varrho} Q_{1}(\tau, v, \lambda(v)) d v, \text { and } B_{\mu}(\tau)=\int_{0}^{\varrho} Q_{2}(\tau, v, \mu(v)) d v .
$$

If $\exists 0<k^{*}<1$ such that $\forall \lambda, \mu \in U$,

$$
\left\|A_{\lambda}-B_{\mu}\right\| \leq k^{*} \mathbf{N}_{(A, B, g, h, \lambda, \mu)},
$$

where

$$
\mathbf{N}_{(A, B, g, h, \lambda, \mu)}=\max \left\{\begin{array}{c}
\|\lambda-\mu\|,\left\|\lambda-A_{\lambda}\right\|+\left\|\mu-B_{\mu}\right\|,\left\|\mu-A_{\lambda}\right\|+\left\|\lambda-B_{\mu}\right\|, \\
\frac{1}{t^{2}}(t+\|\lambda-\mu\|)\left(t\left\|\lambda-A_{\lambda}\right\|+t\left\|\mu-B_{\mu}\right\|+\left\|\lambda-A_{\lambda}\right\| \cdot\left\|\mu-B_{\mu}\right\|\right)
\end{array}\right\},
$$

then, the two nonlinear integral equations defined in (60) have a unique common solution in $U$.
Proof. Define the integral operators $A, B, g, h: U \longrightarrow U$ by

$$
A(\lambda)=A_{\lambda}, B(\mu)=B_{\mu}, g(\mu)=\mu \text { and } h(\lambda)=\lambda .
$$

The NIEs in (60) have a unique common solution iff A $, B, g$, and $h$ have a unique CFP in $U$. Now, we prove that Theorem 10 applies to the integral operators $A, B, g$, and $h$. Then, $\forall \lambda, \mu \in U$, we may have the following four cases arises:

(a) If $\mathbf{N}_{(A, B, g, h, \lambda, \mu)}=\|\lambda-\mu\|$ in (66). Then, by using (62), (65), and (67), we have

$$
\begin{aligned}
\frac{1}{M_{r}(A \lambda, B \mu, t)}-1 & =\frac{d(A \lambda, B \mu)}{t} \leq k^{*} \frac{\mathbf{N}_{(A, B, g, h, \lambda, \mu)}}{t}=k^{*} \frac{\|\lambda-\mu\|}{t} \\
& =k^{*}\left(\frac{1}{M_{r}(h \lambda, g \mu, t)}-1\right) .
\end{aligned}
$$

This implies that

$$
\frac{1}{M_{r}(A \lambda, B \mu, t)}-1 \leq k^{*}\left(\frac{1}{M_{r}(h \lambda, g \mu, t)}-1\right), \text { for } t \gg \theta,
$$

$\forall \lambda, \mu \in U$. Hence, the integral operators $A, B, g$, and $h$ satisfy the conditions of Theorem 10 with $k^{*}=a$ and $b=c$ $=d=0$ in (4). The operators $A, B, g$, and $h$ have a unique CFP, i.e., the unique common solution of the two NIEs (60) in $U$.

(b) If $\mathbf{N}_{(A, B, g, h, \lambda, \mu)}=1 / t^{2}(t+\|\lambda-\mu\|)\left(t\left\|\lambda-A_{\lambda}\right\|+t \| \mu-\right.$ $\left.B_{\mu}\|+\| \lambda-A_{\lambda}\|\cdot\| \mu-B_{\mu} \|\right)$ in (66). Then, by using (62) and (65), we have

$$
\begin{aligned}
\frac{1}{M_{r}(A \lambda, B \mu, t)}-1= & \frac{d(A \lambda, B \mu)}{t} \leq k^{*} \frac{\mathbf{N}_{(A, B, g, h, \lambda, \mu)}}{t} \\
= & k^{*} \frac{1}{t^{3}}(t+\|\lambda-\mu\|)\left(t\left\|\lambda-A_{\lambda}\right\|+t\left\|\mu-B_{\mu}\right\|\right. \\
& \left.+\left\|\lambda-A_{\lambda}\right\| \cdot\left\|\mu-B_{\mu}\right\|\right) .
\end{aligned}
$$

It yields that

$$
\begin{aligned}
\frac{1}{M_{r}(A \lambda, B \mu, t)}-1 \leq & k^{*} \frac{1}{t^{3}}(t+\|h \lambda-g \mu\|)\left(t\left\|h \lambda-A_{\lambda}\right\|+t\left\|g \mu-B_{\mu}\right\|\right. \\
& \left.+\left\|h \lambda-A_{\lambda}\right\| \cdot\left\|g \mu-B_{\mu}\right\|\right),
\end{aligned}
$$

$\forall \lambda, \mu \in U$, and $t \gg \theta$. Now first, we have to simplify the term $M_{o}(h \lambda, g \mu, t) / M_{o}(h \lambda, B \mu, 2 t) * M_{o}(g \mu, A \lambda, 2 t)-1$ by 
using Definition 2 (3) and (62), for $t \gg \theta$,

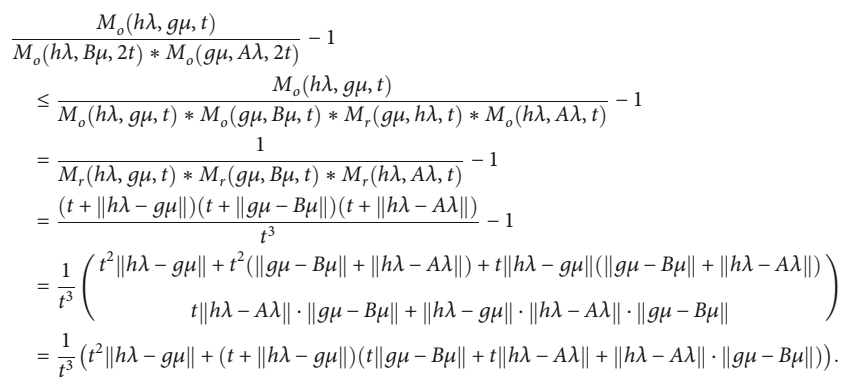

This implies that

$$
\begin{aligned}
& \frac{M_{o}(h \lambda, g \mu, t)}{M_{o}(h \lambda, B \mu, 2 t) * M_{o}(g \mu, A \lambda, 2 t)}-1 \\
& \leq \frac{1}{t^{3}}\left(t^{2}\|h \lambda-g \mu\|+(t+\|h \lambda-g \mu\|)(t\|g \mu-B \mu\|\right. \\
& +t\|h \lambda-A \lambda\|+\|h \lambda-A \lambda\| \cdot\|g \mu-B \mu\|)),
\end{aligned}
$$

$\forall \lambda, \mu \in U$, and $t \gg \theta$. Now, from (71) and (73), we have

$$
\frac{1}{M_{r}(A \lambda, B \mu, t)}-1 \leq k^{*}\left(\frac{M_{o}(h \lambda, g \mu, t)}{M_{o}(h \lambda, B \mu, 2 t) * M_{o}(g \mu, A \lambda, 2 t)}-1\right) \text {, for } t \gg \theta,
$$

$\forall \lambda, \mu \in U$. Hence, the integral operators $A, B, g$, and $h$ satisfy the conditions of Theorem 10 with $k^{*}=b$ and $a=c$ $=d=0$ in (4). The operators $A, B, g$, and $h$ have a unique CFP, i.e., the unique common solution of the two NIEs (60) in $U$.

(c) If $\mathbf{N}_{(A, B, g, h, \lambda, \mu)}=\left\|\lambda-A_{\lambda}\right\|+\left\|\mu-B_{\mu}\right\|$ in (66). Then by using (62), (65), and (67), we have

$$
\begin{aligned}
\frac{1}{M_{r}(A \lambda, B \mu, t)}-1 & =\frac{d(A \lambda, B \mu)}{t} \leq k^{*} \frac{\mathbf{N}_{(A, B, g, h, \lambda, \mu)}}{t} \\
& =k^{*} \frac{\left\|\lambda-A_{\lambda}\right\|+\left\|\mu-B_{\mu}\right\|}{t} \\
& =k^{*}\left(\frac{1}{M_{r}(h \lambda, A \lambda, t)}-1+\frac{1}{M_{r}(g \mu, B \mu, t)}-1\right) .
\end{aligned}
$$

This implies that

$\frac{1}{M_{r}(A \lambda, B \mu, t)}-1 \leq k^{*}\left(\frac{1}{M_{r}(h \lambda, A \lambda, t)}-1+\frac{1}{M_{r}(g \mu, B \mu, t)}-1\right)$, for $t \gg \theta$,

for all $\lambda, \mu \in U$. Hence, the integral operators $A, B, g$, and $h$ satisfy the conditions of Theorem 10 with $k^{*}=c$ and $a=$ $b=d=0$ in (4). The operators $A, B, g$, and $h$ have a unique CFP, i.e., the unique common solution of the two NIEs (60) in $U$. (d) If $\mathbf{N}_{(A, B, g, h, \lambda, \mu)}=\left\|\mu-A_{\lambda}\right\|+\left\|\lambda-B_{\mu}\right\|$ in (66). Then, by using (62), (65), and (67), we have

$$
\begin{aligned}
\frac{1}{M_{r}(A \lambda, B \mu, t)}-1 & =\frac{d(A \lambda, B \mu)}{t} \leq k^{*} \frac{\mathbf{N}_{(A, B, g, h, \lambda, \mu)}}{t} \\
& =k^{*} \frac{\left\|\mu-A_{\lambda}\right\|+\left\|\lambda-B_{\mu}\right\|}{t} \\
& =k^{*}\left(\frac{1}{M_{r}(g \mu, A \lambda, t)}-1+\frac{1}{M_{r}(h \lambda, B \mu, t)}-1\right) .
\end{aligned}
$$

This implies that

$\frac{1}{M_{r}(A \lambda, B \mu, t)}-1 \leq k^{*}\left(\frac{1}{M_{r}(g \mu, A \lambda, t)}-1+\frac{1}{M_{r}(h \lambda, B \mu, t)}-1\right)$, for $t \gg \theta$,

for all $\lambda, \mu \in U$. Hence, the integral operators $A, B, g$, and $h$ satisfy the conditions of Theorem 10 with $k^{*}=d$ and $a=$ $b=c=0$ in (4). The operators $A, B, g$, and $h$ have a unique CFP, i.e., the unique common solution of the two NIEs (60) in $U$

\section{Conclusion}

In this paper, we proved some unique CFP theorems by using the compatible and weakly-compatible four selfmappings in FCM space. We proved the results under the generalized rational contraction conditions in FCM spaces with help of one self-map are continuous. Moreover, we proved some rational contraction results with the weaker condition of self-map continuity. Ultimately, we provide an application of the two NIEs for our theoretical results that have been utilized to prove the existence common solution of the two NIEs to support our main work. This is an illustrative application of how FCM spaces can be used in other integral type operators.

\section{Data Availability}

Data sharing does not apply to this article as no data set were generated or analyzed during the current study.

\section{Conflicts of Interest}

The authors declare that there is no conflict of interest regarding the publication of this paper.

\section{Authors' Contributions}

The authors have equally contributed to the final manuscript.

\section{Acknowledgments}

This work was funded by the Deanship of Scientific Research (DSR), King Abdulaziz University, Jeddah, under Grant no. FP-045-43. 


\section{References}

[1] S. Banach, "Sur les opérations dans les ensembles abstraits et leur application aux équations intégrales," Fundamenta Mathematicae, vol. 3, pp. 133-181, 1922.

[2] S. K. Chatterjea, "Fixed point theorems," Dokladi na B"lgarskata Akademiya na Naukite, vol. 25, pp. 727-730, 1972.

[3] D. Chatterjea, "Generalized contraction principle," International Journal of Mathematics and Mathematical Sciences, vol. 6, no. 1, 94 pages, 1983.

[4] R. Kannan, "Some results on fixed points," Bulletin of the Calcutta Mathematical Society, vol. 60, pp. 71-76, 1968.

[5] M. U. Ali, H. Aydi, and M. Alansari, "New generalizations of set valued interpolative Hardy-Rogers type contractions in bmetric spaces," Journal of Function Spaces, vol. 2021, Article ID 6641342, 8 pages, 2021.

[6] H. Covitz and S. B. Nadler, "Multi-valued contraction mappings in generalized metric spaces," Israel Journal of Mathematics, vol. 8, no. 1, pp. 5-11, 1970.

[7] P. Z. Daffer and H. Kaneko, "Fixed points of generalized contractive multi-valued mappings," Journal of Mathematical Analysis and Applications, vol. 192, no. 2, pp. 655-666, 1995.

[8] H. Kaneko, "Single and multivalued contractions," Bollettino dell'Unione Matematica Italiana, vol. 6, pp. 29-33, 1985.

[9] A. R. Khan, "Properties of fixed point set of a multivalued map," Journal of Applied Mathematics and Stochastic Analysis, vol. 2005, no. 3, pp. 323-331, 2005.

[10] P. Patle, D. Patel, H. Aydi, and S. Radenović, "On $H^{+}$type multivalued contractions and applications in symmetric and probabilistic spaces," Mathematics, vol. 7, no. 2, p. 144, 2019.

[11] L. A. Zadeh, "Fuzzy sets," Information and Control, vol. 8, no. 3, pp. 338-353, 1965.

[12] O. Kramosil and J. Michalek, "Fuzzy metric and statistical metric spaces," Kybernetika, vol. 11, pp. 336-344, 1975.

[13] A. George and P. Veeramani, "On some results in fuzzy metric spaces,” Fuzzy Sets and Systems, vol. 64, no. 3, pp. 395-399, 1994.

[14] M. Grabiec, "Fixed points in fuzzy metric spaces," Fuzzy Sets and Systems, vol. 27, no. 3, pp. 385-389, 1988.

[15] V. Gregori and A. Sapena, "On fixed-point theorems in fuzzy metric spaces," Fuzzy sets and Systems, vol. 125, no. 2, pp. 245-252, 2002.

[16] O. Hadzic and E. Pap, "A fixed point theorem for multivalued mappings in probabilistic metric spaces and an application in fuzzy metric spaces," Fuzzy Sets and Systems, vol. 127, no. 3, pp. 333-344, 2002.

[17] M. Imdad and J. Ali, "Some common fixed point theorems in fuzzy metric spaces," Mathematical Communications, vol. 11, pp. 153-163, 2006.

[18] S. U. Rehman, R. Chinram, and C. Boonpok, "Rational type fuzzy-contraction results in fuzzy metric spaces with an application," Journal of Mathematics, vol. 2021, Article ID 6644491, 13 pages, 2021.

[19] B. D. Pant and S. Chauhan, "Common fixed point theorems for two pairs of weakly compatible mappings in menger spaces and fuzzy metric spaces," Scientific Studies and Research Series Mathematics and Informatics, vol. 21, pp. 81-96, 2011.

[20] F. Kiyani and A. Amini-Haradi, "Fixed point and endpoint theorems for set-valued fuzzy contraction maps in fuzzy metric spaces," Fixed Point Theory and Applications, vol. 2011, no. $1,2011$.
[21] Z. Sadeghi, S. M. Vaezpour, C. Park, R. Saadati, and C. Vetro, "Set-valued mappings in partially ordered fuzzy metric spaces," Journal of Inequalities and Applications, vol. 2014, no. $1,2014$.

[22] L. Huang and X. Zhang, "Cone metric spaces and fixed point theorems of contractive mappings," Journal of Mathematical Analysis and Applications, vol. 332, no. 2, pp. 1468-1476, 2007.

[23] M. Abbas and G. Jungck, "Common fixed point results for noncommuting mappings without continuity in cone metric spaces," Journal of Mathematical Analysis and Applications, vol. 341, no. 1, pp. 416-420, 2008.

[24] N. B. Huy and T. D. Thanh, "Fixed point theorems and the Ulam-Hyers stability in non-Archimedean cone metric spaces," Journal of Mathematical Analysis and Applications, vol. 414, no. 1, pp. 10-20, 2014.

[25] S. Radenović, "Common fixed points under contractive conditions in cone metric spaces," Computers and Mathematics with Applications, vol. 58, no. 6, pp. 1273-1278, 2009.

[26] M. Rangamma and K. Prudhvi, "Common fixed points under contractive conditions for three maps in cone metric spaces," Bulletin of Mathematical Analysis and Applications, vol. 4, pp. 174-180, 2012.

[27] H. Aydi, E. Karapinar, and W. Shatanawi, "Coupled fixed point results for $(\psi, \varphi)$-weakly contractive condition in ordered partial metric spaces," Computers and Mathematics with Applications, vol. 62, no. 12, pp. 4449-4460, 2011.

[28] D. Turkoglu and M. Abuloha, "Cone metric spaces and fixed point theorems in diametrically contractive mappings," Acta mathematica sinica, vol. 26, no. 3, pp. 489-496, 2010.

[29] T. Öner, M. B. Kandemir, and B. Tanay, "Fuzzy cone metric spaces," Journal of Nonlinear Sciences and Applications, vol. 8, no. 5, pp. 610-616, 2015.

[30] S. Ur Rehman and H. X. Li, "Fixed point theorems in fuzzy cone metric spaces," Journal of Nonlinear Sciences and Applications, vol. 10, no. 11, pp. 5763-5769, 2017.

[31] S. U. Rehman and H. Aydi, "Rational fuzzy cone contractions on fuzzy cone metric spaces with an application to Fredholm integral equations," Journal of Function Spaces, vol. 2021, Article ID 5527864, 13 pages, 2021.

[32] Saif Ur Rehman, Ihsan Khan, Hayat Ullah, Shamoona Jabeen, and Fatima Abbas, "Common fixed point theorems for compatible and weakly compatible maps in fuzzy cone metric spaces," Annals of Fuzzy Mathematics and Informatics, vol. 19, no. 1, pp. 1-19, 2020.

[33] S. Jabeen, S. Ur Rehman, Z. Zheng, and W. Wei, "Weakly compatible and quasi-contraction results in fuzzy cone metric spaces with application to the Urysohn type integral equations," Advances in Differential Equations, vol. 2020, no. 1, article 280, 2020.

[34] B. Schweizer and A. Sklar, "Statical metric spaces," Pacific Journal of Mathematics, vol. 10, pp. 314-334, 1960. 